\title{
COMPARATIVE STUDY OF IV DEXMEDETOMIDINE AND FENTANYL ON HEMODYNAMIC RESPONSE, SEDATION, ANALGESIA AND ANXIOLYSIS IN SURGERIES REQUIRING LOCAL ANAESTHESIA WITH INTRAVENOUS SEDATION
}

\author{
Anam Amin Kazi'1, Aparna Amar Ruparel2, Shreyas Rao ${ }^{3}$, Akhil Bhalla4 4 , Amit Nagpal ${ }^{5}$
}

1 Junior Resident, Department of Anaesthesia, Dr. D. Y. Patil Medical College and Hospital, Nerul, Navi Mumbai, Maharashtra, India. 2Junior Resident, Department of Anaesthesia, Dr. D. Y. Patil Medical College and Hospital, Nerul, Navi Mumbai, Maharashtra, India. 3Junior Resident, Department of Anaesthesia, Dr. D. Y. Patil Medical College and Hospital, Nerul, Navi Mumbai, Maharashtra, India. ${ }_{4}^{4}$ Junior Resident, Department of Anaesthesia, Dr. D. Y. Patil Medical College and Hospital, Nerul, Navi Mumbai, Maharashtra, India. ${ }_{5}^{5}$ Assistant Professor, Department of Anaesthesia, Dr. D. Y. Patil Medical College and Hospital, Nerul, Navi Mumbai, Maharashtra, India. ABSTRACT

\section{BACKGROUND}

LA with IV sedation is referred to as twilight anaesthesia. The choice and route of anaesthesia administration is paramount to the patient's overall surgical experience. The qualities of a good sedative or anaesthetic agent include sedation, anxiolysis, analgesia and amnesia. This study was planned to compare effects of IV Dexmedetomidine and IV Fentanyl that lead to changes in hemodynamic response.

Aims and Objectives

1. To compare Dexmedetomidine and Fentanyl in surgery requiring Local Anaesthetic with respect to IV sedation, analgesia, anxiolysis and hemodynamic changes.

2. To compare post-operative recovery time.

3. To compare side effects of the drugs.

4. To compare patient satisfaction.

\section{MATERIALS AND METHODS}

A total of 60 patients in the age group of 18-60 yrs. of either sex undergoing elective surgery requiring local anaesthetic agent with IV sedation, categorized under ASA 1, 2 were included. These patients were divided into 2 groups of 30 patients each. Sample size was taken for convenience. Group A patients received Dexmedetomidine $1 \mathrm{mcg} / \mathrm{kg}$ IV loading dose over 10 mins followed by 0.2 $\mathrm{mcg} / \mathrm{Kg} / \mathrm{hr}$ IV infusion. Group B patients received fentanyl $1 \mathrm{mcg} / \mathrm{Kg}$ IV over $10 \mathrm{mins}$ followed by $0.5 \mathrm{mcg} / \mathrm{Kg} / \mathrm{hr}$ IV infusion. Heart rate mean arterial blood pressure, respiratory rate, $\mathrm{SpO}_{2}$ were recorded every 10 mins. Tramadol $2 \mathrm{mg} / \mathrm{Kg}$ slow IV was given as rescue analgesic and the surgeon used an additional dose of local anaesthetic. Ramsay sedation score was assessed. Visual Analogue Scale of more than 3, analgesia was provided with $75 \mathrm{mg}$ slow IV diclofenac. Aldrete score was assessed in recovery room.

\section{RESULTS}

From statistical data derived, it was observed that dexmedetomidine provided more sedation (RSS: $2 \pm 0.6$ with fentanyl, RSS: $3 \pm 1.3$ with dexmed), anxiolysis and hemodynamic stability as compared to fentanyl. For analgesia, results were comparable for both groups (5.9-6.9 with dexmed, 7.4-8.3 with fentanyl). Bradycardia (13.3\% in dexmed and 3.3\% in fentanyl) and hypotension (6.6\% in dexmed and $3.3 \%$ in fentanyl) were more common with dexmedetomidine than with fentanyl. Itching was $30 \%$ more common with fentanyl while only $3.3 \%$ in dexmed.

\section{CONCLUSION}

Dexmedetomidine provides better sedation and anxiolysis by decreased heart rate, systolic BP, diastolic BP, mean BP. Analgesic effect is comparable in both groups. Dexmedetomidine causes less nausea and vomiting, shorter recovery period and a higher level of patient and surgeon's satisfaction than fentanyl.

\section{KEY WORDS}

Dexmedetomidine, Fentanyl, Surgeries Under Local Anaesthesia, Analgesia Score, Sedation Score

HOW TO CITE THIS ARTICLE: Kazi AA, Ruparel AA, Rao S, et al. Comparative study of IV dexmedetomidine and fentanyl on hemodynamic response, sedation, analgesia and anxiolysis in surgeries requiring local anaesthesia with intravenous sedation. J. Evolution Med. Dent. Sci. 2019;8(11):783-787, DOI: 10.14260/jemds/2019/172

'Financial or Other Competing Interest': None.

Submission 24-01-2019, Peer Review 28-02-2019,

Acceptance 06-03-2019, Published 18-03-2019.

Corresponding Author:

Dr. Anam Amin Kazi,

\#104, Jubilee Nivas Abad Baug,

Off Yari Road, Andheri West,

Mumbai-400061, Maharashtra, India.

E-mail: anamamink@gmail.com

DOI: $10.14260 /$ jemds $/ 2019 / 172$

\section{BACKGROUND}

Local Anaesthesia with intravenous sedation is referred to as twilight anaesthesia. ${ }^{1}$ It provides excellent analgesia to the patient, with minimum use of anaesthesia drugs also providing suitable conditions for the surgeon to operate.

Anxiety related to surgery under local anaesthesia can lead to severe consequences during the surgery. Hence most patients demand a form of sedation along with the local anaesthetic. It may be in the form of intravenous, oral, intramuscular or inhalational. Adequate depth of sedation 
and analgesia to allay anxiety and any pain stimulus associated with the surgery. However, the main problem with most of the sedatives and opioids is the associated respiratory depression. ${ }^{2}$

The qualities of a good sedative or anaesthetic agent include sedation, anxiolysis, analgesia and amnesia.

Sedation refers to the depression of the level of consciousness. Analgesia is the absence of pain. Amnesia is the loss of memory.

Dexmedetomidine is a new drug with highly selective agonistic action at alpha-2 adrenergic receptor. The consequence is a reduction in noradrenergic neurotransmitter release and depression of adrenergic pathways. $^{3}$ It produces sedation in a manner similar to sleep. It activates presynaptic alpha 2 receptors in the spinal cord, activation of the descending inhibitory pain pathway and by inhibiting the release of substance P. Dexmedetomidine is indicate for sedation of intubated or mechanically ventilated post- surgically in the ICU. ${ }^{3}$ It is also used for sedation of nonintubated patients prior to or during surgery via continuous infusion. It includes monitored anaesthesia care with the use of local anaesthetics for dentistry, ENT surgeries, ophthalmic surgeries, MRI, CT. It has the ability to sedate and provide analgesia while maintaining patient arousability and respiratory function. ${ }^{3}$

Fentanyl is a synthetic opioid agonist with excellent analgesic properties. It can blunt circulatory changes in response to any surgical stimulation. ${ }^{2}$

Hence this study is planned to compare effects of intravenous dexmedetomidine and intravenous fentanyl with respect to sedation, analgesia, anxiolysis leading to changes in hemodynamic response.

\section{MATERIALS AND METHODS}

\section{Subjects}

All patients in the age group of 18-60 years undergoing elective surgeries requiring LA with IV sedation, categorized under ASA I and II.

\section{Study Design}

This non randomized, control trail study.

\section{Inclusion Criteria}

- $\quad$ Patients of either sex undergoing surgeries requiring LA with IV sedation.

- Patients in the age group of 18-60 years.

- $\quad$ Patients under ASA I and II.

\section{Exclusion Criteria}

- Non-consenting patients.

- Pregnant patients.

- Lactating patients.

- Emergency cases.

- Patients with associated uncontrolled systemic diseases.

\section{Sample Size}

60 patients. Sample size was taken for convenience.

\section{Study Procedure}

Informed consent was taken from each of the patients for the study. This non randomized, control trail study sample size estimation done by conveniences.
Patients between the age group of 18 to 60 years, scheduled for elective surgery under local anaesthesia like tympanoplasty, ossiculoplasty, modified radical mastoidectomy etc. were included in the study.

Patients were divided into 2 groups of 30 each. Group A received Dexmedetomidine and Group B received Fentanyl for sedation in the course of the surgery.

Patients were premedicated with $0.2 \mathrm{mg}$ Glycopyrrolate and $4 \mathrm{mg}$ ondansetron.

The drugs were prepared by another anaesthesiologist who was not involved in the study and diluted up to $50 \mathrm{ml}$ volume. The anaesthesiologist performing the study and recording data was also blind to the patient group assignment.

Basic monitors including ECG, non-invasive blood pressure, pulse oximetry were attached and baseline vitals were recorded.

Oxygen was given at the rate of $2 \mathrm{~L} / \mathrm{min}$ via nasal prongs.

Ramsay Sedation Score was assessed every 10 mins.

Local Anaesthesia was given by the operating surgeon using lidocaine $2 \%$ plus adrenaline 1:200000. The surgeon was also unaware of the study group the patient belonged to.

Group A patients received Dexmedetomidine $1 \mathrm{mcg} / \mathrm{kg}$ iv loading dose over $10 \mathrm{mins}$ followed by $0.2 \mathrm{mcg} / \mathrm{kg} / \mathrm{hr}$ iv infusion.

Group B patients received Fentanyl $1 \mathrm{mcg} / \mathrm{kg}$ IV over 10 mins followed by $0.5 \mathrm{mcg} / \mathrm{kg} / \mathrm{hr}$ IV infusion

Ramsay Sedation score was assessed. Target sedation level was defined as RSS $>=3$. If RSS was $<3$ additional sedation was given with midazolam $0.03-0.05 \mathrm{mg} / \mathrm{kg}$ IV.

The surgeon performed the surgery under local anaesthesia.

In case the patient complained of pain during the surgery, inj. tramadol $2 \mathrm{mg} / \mathrm{kg}$ slow IV was given as rescue analgesia and an additional dose of the local anaesthetic was given by the surgeon.

Heart rate, mean arterial blood pressure, respiratory rate, peripheral oxygen saturation were recorded every 10 mins.

Infusion of the test drug was stopped just before surgical closure by the surgeon.

After completion of the surgery, patients were shifted to the recovery room.

Post- operative pain was assessed using Visual Analogue Scale (0-10). If VAS was $>3$, analgesia was provided with diclofenac $75 \mathrm{mg}$ slow IV.

Aldrete score was assessed in recovery room every 10 mins, till a score of 10 was achieved. Time to which a score of 10 was achieved was noted. This was the criteria to shift the patient to the ward.

Patients were asked to rate their experience with the sedation and analgesia received during the surgery using a 7 point Likert verbal rating scale just before shifting the patient to the ward. Acceptable satisfaction score of the patient was 5-7.

All the adverse events including bradycardia, hypotension, respiratory depression, oxygen desaturation, nausea, vomiting were recorded.

\section{Scores Used in the Study are Mentioned Below A) Ramsay Sedation Score}

1. Anxious, agitated, restless.

2. Cooperative, oriented, tranquil. 
3. Responds to command.

4. Asleep but has a brisk response to light glabellar tap or loud auditory stimulus.

5. Asleep, has a sluggish response to a light glabellar tap or loud auditory stimulus.

6. Asleep, no response.

\section{B) Likert Scale}

1. Extremely dissatisfied.

2. Dissatisfied.

3. Slightly dissatisfied.

4. Undecided.

5. Slightly satisfied.

6. Satisfied.

7. Extremely satisfied.

\section{C) Modified Aldrete Scale.}

\begin{tabular}{|c|c|}
\hline Item & Answer Choices (points) \\
\hline 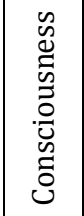 & $\begin{array}{l}\text { Fully wake 2) } \\
\text { Arousable (1) } \\
\text { Not responding (0) }\end{array}$ \\
\hline$\stackrel{D}{:}$ & $\begin{array}{l}\text { Able to move four extremities on command (2) } \\
\text { Able to move two extremities on command (1) } \\
\text { Able to move } 0 \text { extremities on command ( } 0)\end{array}$ \\
\hline 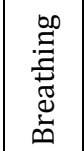 & $\begin{array}{l}\text { Able to breathe deeply (2) } \\
\text { Dyspnoea (1) } \\
\text { Apnoea (0) }\end{array}$ \\
\hline 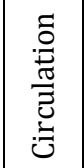 & $\begin{array}{l}\text { Systemic BP } \neq 20 \% \text { of the preanesthetic level ( } 2 \text { ) } \\
\text { Systemic BP between } 20 \% \text { and } 49 \% \text { of the } \\
\text { preanesthetic level ( } 1 \text { ) } \\
\text { Systemic BP } \neq 50 \% \text { of the preanesthetic level (0) }\end{array}$ \\
\hline$\frac{\tilde{0}}{0}-$ & $\begin{array}{l}\text { Normal (2) } \\
\text { Pale, jaundiced, blotchy (1) } \\
\text { Cyanotic (0) }\end{array}$ \\
\hline 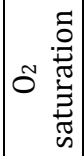 & $\begin{array}{l}\text { Maintaining } \mathrm{O}_{2} \text { saturation }>90 \% \text { on room air }(2) \\
\text { Needs inhalation to maintain } \mathrm{O}_{2} \text { saturation }>90 \%(1) \\
02 \text { saturation }<90 \% \text { despite } \mathrm{O}_{2} \text { supplementation (0) }\end{array}$ \\
\hline
\end{tabular}

\section{D) Visual Analogue Scale}

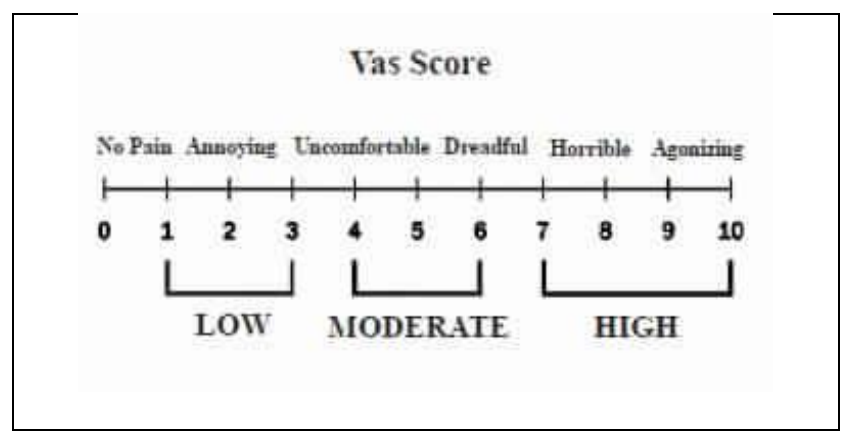

Patients Were Monitored as Pre-Defined Time Interval as Follows

- $\quad$ T0- baseline, prior to the start of premedication

- T1- start of loading dose infusion

- $\quad \mathrm{T} 2-$ at the end of loading dose infusion after 10 mins

- $\quad$ T3- just after infiltration of local anaesthetic drug at the site of the surgery

- T4- 5 minutes after $\mathrm{t} 3$

- Every 10 minutes thereafter, till the end of the procedure and in the recovery room.

\section{Statistical Analysis}

Demographic data and baseline measurements were used in group differences and were tested using Fisher's exact test \& Independent sample t-test, respectively. The software used was Sigma Stat 3.5. P value $<0.001$ was taken as significant.

\section{RESULTS}

Demographic information: A total of 60 patients who underwent surgery requiring LA with IV sedation were enrolled for the study and were randomly divided into two groups. The demographic characteristics in both the groups exhibited marked similarities with respect to sex, age, weight, height and did not show any statistically significant difference ( $p>0.05)$.

\begin{tabular}{|c|c|c|c|c|}
\hline $\begin{array}{c}\text { Sex Count } \\
(\%)\end{array}$ & \multicolumn{2}{|c|}{ Dexmedetomidine } & \multicolumn{2}{c|}{ Fentanyl } \\
\hline & Count & Percentage & Count & Percentage \\
\hline Male & 16 & 53.3 & 18 & 60 \\
\hline Female & 14 & 46.7 & 12 & 40 \\
\hline
\end{tabular}

\begin{tabular}{|c|c|c|}
\hline Age & Dexmedetomidine & Fentanyl \\
\hline Mean \pm SD & $32.7 \pm 9.8$ & $31.3 \pm 8.6$ \\
\hline Range & $18-56$ & $16-55$ \\
\hline
\end{tabular}

\begin{tabular}{|c|c|c|}
\hline Weight & Dexmedetomidine & Fentanyl \\
\hline Mean \pm SD & $59.53 \pm 10.07$ & $59.06 \pm 6.8$ \\
\hline Range & $36-83$ & $45-70$ \\
\hline
\end{tabular}

\begin{tabular}{|c|c|c|}
\hline Height & Dexmedetomidine & Fentanyl \\
\hline Mean \pm SD & $5.4 \pm 0.2$ & $5.5 \pm 0.2$ \\
\hline Range & $5.0 \pm 5.7$ & $5.0 \pm 5.8$ \\
\hline
\end{tabular}

\section{Operation Proposed}

\begin{tabular}{|c|c|c|c|c|}
\hline & \multicolumn{2}{|c|}{ Dexmedetomidine } & \multicolumn{2}{c|}{ Fentanyl } \\
\hline $\begin{array}{c}\text { Operation } \\
\text { Proposed }\end{array}$ & Count & Percentage & Count & Percentage \\
\hline MRM & 5 & 16.7 & 5 & 16.7 \\
\hline Ossiculoplasty & 2 & 16.7 & 3 & 10.0 \\
\hline T-plasty & 23 & 76.7 & 22 & 73.3 \\
\hline p Value & 0.001 & & & \\
\hline
\end{tabular}

\begin{tabular}{|c|c|c|c|c|c|}
\hline & \multicolumn{2}{|c|}{ Dexmedetomidine } & \multicolumn{2}{|c|}{ Fentanyl } & P-value \\
\hline & Mean & SD & Mean & SD & 2 Sample \\
\hline Pulse & 72 & 8.1 & 75 & 6.3 & 0.002 \\
\hline $\mathrm{RR}$ & 17 & 2.3 & 15 & 2.4 & 0.014 \\
\hline SBP & 115 & 6.9 & 120 & 5.4 & 0.022 \\
\hline DBP & 69 & 7.4 & 75 & 4.9 & 0.011 \\
\hline MAP & \multicolumn{2}{|c|}{85} & \multicolumn{2}{|c|}{90} & 0.015 \\
\hline Sedation & 3 & 1.3 & 2 & 0.6 & 0.004 \\
\hline
\end{tabular}




\section{DISCUSSION}

Monitored anaesthesia care is a technique of combining local anaesthesia with parenteral drugs for sedation and analgesia. ${ }^{4}$ Fentanyl is a versatile drug, but may have the risk of losing airway control, hypoxia and hypotension with higher doses. Recently use of highly specific alpha 2 agonists such as dexmedetomidine developed for perioperative period as well as sedation and analgesia. It also leads to induced hypotension, hence providing a bloodless surgical field. ${ }^{5}$

In this study, Fentanyl and Dexmedetomidine infusions were used and a comparative study was performed between the two with respect to sedation, analgesia, anxiolysis as well as patient's satisfaction and post-operative residual drug effects for surgeries conducted under local anaesthesia. It was found that mean Ramsay sedation score was significantly more in dexmedetomidine group (RSS: $3 \pm 1.3$ ) than in fentanyl group (RSS: $2 \pm 0.6) .7$ Dexmedetomidine can provide more sedation when compared to fentanyl for surgery requiring LA with IV sedation. Lowered heart rate, no respiratory depression, easy awakening, can be achieved by the use of dexmedetomidine. ${ }^{6}$

The study did not show any significant difference on statistical comparison with respect to sex, age, weight, height.

It was found that $1 \mathrm{mcg} / \mathrm{kg}$ of loading dose with maintenance of $0.2 \mathrm{mcg} / \mathrm{kg}$ for dexmedetomidine provided higher Ramsay Sedation Score (RSS between 3-5) when compared to fentanyl (RSS between 1-2).

Rescue Sedation to achieve target sedation level (Ramsay score of $>3$ ) was required by significantly higher number of patients in fentanyl group (20\% patients) as compared to dexmedetomidine group $(0 \%$ patients). A progressive increase in sedation occurs with increasing dose of dexmedetomidine. Dexmedetomidine bolus dose was used 1 $\mathrm{mcg} / \mathrm{kg}$ over 10 mins followed by continuous IV infusion 0.2 $\mathrm{mcg} / \mathrm{kg} / \mathrm{hr}$. RSS was maintained $>3$ throughout intraoperative period and rescue sedation was not required in almost all cases of this group. Equipotent doses of fentanyl in the other group $(0.5 \mathrm{mcg} / \mathrm{kg} / \mathrm{hr}$ maintenance infusion) could not achieve similar results rendering patients fully awake and aware of surroundings.

Intraoperative rescue analgesic requirement was almost same in both groups. VAS score was slightly lower for dexmedetomidine group (5.9-6.9) than fentanyl group (7.48.3) indicating more potent analgesic effect of fentanyl.7

Dexmedetomidine patients showed a significant reduction in heart rate which occurred most commonly after loading dose which achieved peak effect at 10-30 mins of maintenance infusion. ${ }^{3}$ This occurs due to 1 ) lower basal heart rate from increased vagal tone. 2) High doses and decreased circulating levels of norepinephrine. 3) The baroreceptor response of high vascular tone that occurs with the bolus. A negative chronotropic effect was profoundly exhibited by dexmedetomidine approximately 30-35 mins after starting bolus infusion, after which the heart rate remained stable in the range of $56-70 / \mathrm{min}$ in both the groups. ${ }^{4}$

Mean arterial pressure decreased from the baseline in both the groups with a maximum decline of MAP at 30-50 mins after starting drug infusion. Post operatively, HR and MAP remained stable. The decrease in HR caused by alpha -2 agonist can be explained on the basis of their central action whereby they decrease sympathetic outflow and nor- epinephrine release, whereas in fentanyl group, it can be due to analgesic effect. ${ }^{2}$

Intraoperative bleeding was significantly less in dexmedetomidine group as compared to fentanyl group. Controlled hypotension effectively reduced surgical blood loss and improved surgical conditions. This occurs by a decreased heart rate, systolic, diastolic and mean blood pressure. Hemodynamic attenuation produced by dexmedetomidine, resulting from diminished sympathetic outflow by central alpha 2 adrenoceptor stimulation may contribute to reduced bleeding. ${ }^{8}$

Dexmedetomidine produces sedation by decreasing the sympathetic nervous system activity, resulting in a calm patient an easily arousable. Its additional analgesic effect accounts for greater patient satisfaction rate which was assessed by Likert's scale (Median Likert scale for dexmedetomidine was 7, while for fentanyl group it was 4).7

Surgeons also experienced bloodless surgical field by the use of dexmedetomidine group, and less interruption of surgery due to better analgesic effect, hence more surgical satisfaction.

Comparison between Dexmedetomidine and fentanyl were compared, significant difference had been noted regarding the time required to shift the patient to the ward postoperatively from the recovery. All the patients in dexmedetomidine group had modified Aldrete score of 10 within 20-30 mins after shifting to recovery room (mean \pm SD; 28.67 \pm 7.3 ), fentanyl had modified Aldrete score of 10 within 50-60 mins., after shifting to recovery room (Mean \pm SD; $65.7 \pm 15) .^{7}$

\section{CONCLUSION}

The side effects of both the groups exhibited the following

1. Bradycardia in dexmedetomidine occurred in $13.3 \%$ and fentanyl group $3.3 \%$. This was treated by $0.6 \mathrm{mg} \mathrm{IV}$ Atropine.

2. Nausea occurred in $3.3 \%$ of Dexmedetomidine and Fentanyl $16.6 \%$.

3. Hypotension occurred only in $6.6 \%$ of dexmedetomidine group and $3.3 \%$ of Fentanyl group.

4. Itching was significantly higher in fentanyl group (30\%) and Dexmedetomidine group (3.3\%).

\section{REFERENCES}

[1] O'Donnell JM, Bragg K, Sell S. Procedural sedation: safely navigating the twilight zone. Nursing 2003;33(4):36-41, 44, quiz 45.

[2] McClain $\mathrm{DA}_{2}$ Hug CC Jr. Intravenous fentanyl kinetics. Clinical Pharmacology and Therapeutics 1980;28(1):106-14.

[3] Chrysostomou C, Schmitt CG. Dexmedetomidine: sedation, analgesia and beyond. Expert Opinion on Drug Metabolism \& Toxicology 2008;4(5):619-27.

[4] Alhashemi JA. Dexmedetomidine vs midazolam for monitored anaesthesia care during cataract surgery. British Journal of Anaesthesia 2006;96(6):722-6.

[5] Arain SR, Ebert TJ. The efficacy, side effects, and recovery characteristics of dexmedetomidine versus propofol when used for intraoperative sedation. Anesthesia \& Analgesia 2002;95(2):461-6. 
[6] Yu C, Li S, Deng F, et al. Comparison of dexmedetomidine/fentanyl with midazolam/fentanyl combination for sedation and analgesia during tooth extraction. International Journal of Oral and Maxillofacial Surgery 2014;43(4):1148-53.

[7] Schulte-Tamburen AM, Scheier J, Briegel J, et al. Comparison of five sedation scoring systems by means of auditory evoked potentials. Intensive Care Medicine 1999;25(4):377-82.
[8] Ayoglu H, Yapakci O, Ugur MB, et al. Effectiveness of dexmedetomidine in reducing bleeding during septoplasty and tympanoplasty operations. Journal of Clinical Anesthesia 2008;20(6):437-41. 